\title{
Estimativas de parâmetros genéticos para características de crescimento em ovinos da raça Somalis Brasileira
}

\author{
Genetic parameters estimates for growth traits in Somalis Brasileira hair sheep breed
}

\author{
Ana Fabrícia Braga Magalhães ${ }^{I}$ Raimundo Nonato Braga Lobo ${ }^{I I}$ Olivardo Facó ${ }^{I I}$
}

\section{RESUMO}

\begin{abstract}
Objetivou-se estimar parâmetros genéticos para características de crescimento em ovinos da raça Somalis Brasileira. Os dados utilizados neste estudo foram provenientes do rebanho da Embrapa Caprinos e Ovinos, localizado na cidade de Sobral - CE. Os dados continham registros de 1.120 animais no arquivo de pedigree. As características analisadas foram: peso ao nascer $(P N)$, peso ao desmame (PD), ganho de peso do nascimento ao desmame (GND) e peso adulto (PA). As estimativas dos componentes de (co)variâncias e os parâmetros genéticos foram obtidos pelo método da Máxima Verossimilhança Restrita não Derivativa (DFREML), utilizando o programa MTDFREML. Nas análises unicaracterística, as herdabilidades foram de magnitude baixa a moderada. As magnitudes das herdabilidades maternas foram todas superiores às herdabilidades diretas. As herdabilidades das análises multicaracterísticas entre $P N, P D e$ GND superaram as análises unicaracterísticas. As correlações genéticas apresentaram grande variação quanto aos seus valores.
\end{abstract}

Palavras-chave: análise multicaracterística, correlação genética, herdabilidade, peso corporal.

\section{ABSTRACT}

The aim of this study was to estimate genetic parameters for growth traits in Somalis Brasileira hair sheep breed. The data used in this study were from the flock of Embrapa Caprinos and Ovinos, located in the city of Sobral-CE, Brazil. Data contained records of 1,120 animals in the pedigree file. The analyzed traits were birth weight $(P N)$, weaning weight $(P D)$, weight gain from birth to weaning $(G N D)$ and adult weight (PA). Estimates of (co)variances and genetic parameters were obtained by Derivative Free Restricted Maximum Likelihood Method (DFREML) using the MTDFREML software. The heritabilities were low to moderate in univariate analysis. The maternal heritabilities were higher than direct heritabilities. The heritabilities in multivariate analysis exceeded those in univariate analysis. The values of genetic correlations varied widely.

Key words: multivariate analysis, genetic correlation, heritability, body weight.

\section{INTRODUÇÃO}

A raça Somalis Brasileira possui pelagem branca, sem lã, com cabeça e pescoço pretos ou vermelhos e aptidão para produção de carne e pele. SILVA et al. (1986) afirmaram que a raça possui um baixo índice de mortalidade, associado ao bom desempenho reprodutivo, o que a caracteriza com excelente adaptabilidade à região nordestina.

Constituem como principais informações para avaliação genética de ovinos as mensurações dos pesos corporais em intervalos de tempo determinados durante a fase de crescimento (REGO NETO et al., 2010). Os pesos em determinadas idades apresentam-se como características diferentes, geralmente correlacionadas, o que torna fundamental conhecer a magnitude e a direção de tais correlações, uma vez que a seleção de uma delas poderá promover mudanças nas demais (SARMENTO et al., 2006). Para determinar adequadas estratégias de melhoramento genético, é necessário o conhecimento de parâmetros genéticos

'Departamento de Zootecnia, Faculdade de Ciências Agrárias e Veterinárias (FCAV), Universidade Estadual Paulista "Júlio de Mesquita Filho" (UNESP), 14884-900, Jaboticabal, SP, Brasil. E-mail: anafabriciabm@yahoo.com.br. Autor para correspondência.

"Embrapa Caprinos e Ovinos, Sobral, CE, Brasil. 
para características de crescimento em várias idades, tais como as herdabilidades e as correlações genéticas entre elas (BEHZADI et al., 2007). As estimativas dos parâmetros genéticos podem ser realizadas por meio de análises unicaracterística ou multicaracterísticas. A escolha do melhor modelo, segundo SARMENTO et al. (2006), dependerá do resultado final obtido para as herdabilidades e correlações genéticas. Obviamente que os modelos unicaracterística não permitem a obtenção de correlações genéticas.

É importante salientar que, no Brasil, ainda há poucas estimativas de parâmetros genéticos para ovinos. Estudos separando os efeitos genéticos aditivos direto e materno para características de crescimento são ainda em menor número (SARMENTO et al., 2006). Para a raça Somalis Brasileira, essas estimativas são praticamente inexistentes, com apenas um trabalho encontrado na literatura, realizado por SILVA et al. (1994), utilizando o modelo reprodutor. Portanto, objetivouse, com este trabalho, estimar parâmetros genéticos para características de crescimento em ovinos da raça Somalis Brasileira, sob modelo animal.

\section{MATERIAL E MÉTODOS}

Os dados utilizados neste estudo foram provenientes de ovinos da raça Somalis Brasileira, do Núcleo de Conservação da Embrapa Caprinos e Ovinos, localizada na cidade de Sobral, região norte do estado do Ceará, controlado dentro do Programa de Melhoramento Genético de Caprinos e Ovinos de Corte - GENECOC. As características analisadas foram: peso ao nascer (PN), peso ao desmame (PD), ganho de peso do nascimento ao desmame (GND) e peso adulto (PA). Os dados continham registros de 1.120 animais no arquivo de pedigree.

Para análise de PN, PD e GND, os animais foram agrupados em grupos de contemporâneos, constituídos por animais pertencentes ao mesmo tipo de nascimento, estação de nascimento, ano de nascimento e mesmo sexo. Para análise do peso adulto das matrizes (PA), as fêmeas foram agrupadas em grupos de contemporâneos, formados por animais com mesmo ano e estação de pesagem. Cada grupo contemporâneo foi composto de pelo menos quatro animais. Previamente, as características foram analisadas utilizando o procedimento MIXED do programa SAS (SAS INSTITUTE INC, 1996), para definição dos modelos fixos. Para cada característica, foram avaliados vários modelos lineares, com a inclusão de efeitos agrupados em grupos de contemporâneos ou analisando todos os efeitos individualmente e suas interações. Os critérios para a escolha do modelo fixo que melhor se ajustou aos dados foram o Logaritmo da Função de Máxima Verossimilhança Restrita, o Critério de Informação de Akaike e o Critério de Informação Bayesiano de Schwarz. Assim, os efeitos fixos utilizados foram grupo de contemporâneos e idade da mãe ao parto para PN, PD e GND, além da covariável idade ao desmame para PD e GND (efeito linear); para PA, o modelo continha o grupo de contemporâneos e a covariável idade do animal no dia da pesagem (efeito linear e quadrático).

As estimativas dos componentes de (co) variâncias e os parâmetros genéticos foram obtidos pelo método da Máxima Verossimilhança Restrita não Derivativa (DFREML), utilizando o programa MTDFREML (BOLDMAN et al., 1995).

O modelo geral para os pesos corporais e os ganhos de peso, matricialmente, segue:

$\mathrm{Y}=\mathrm{X} \beta+\mathrm{Z}_{1} \mathrm{a}+\mathrm{Z}_{2} \mathrm{~m}+\mathrm{Z}_{3} \mathrm{pe}+\varepsilon$

em que $Y$ é o vetor de observações do animal; $\beta$, o vetor de efeitos fixos no modelo, associados com a matriz de incidência $X$; a, o vetor dos efeitos genéticos diretos, associados com a matriz de incidência $Z_{1} ; m$, o vetor dos efeitos genéticos maternos, associado com a matriz de incidência $\mathrm{Z}_{2}$; pe, o vetor dos efeitos de ambiente permanente materno, associado com a matriz de incidência $\mathrm{Z}_{3}$; e $\varepsilon$, o vetor de resíduos aleatórios. Ressalta-se que o componente $\mathrm{Z}_{2} \mathrm{~m}$ não estava presente na análise para peso adulto e que o componente $Z_{3}$ pe refere-se aos efeitos de ambiente permanente do animal.

As covariâncias entre os efeitos genético aditivo direto e materno foram consideradas como zero, segundo recomendação de SCHAEFFER (1996). Isso se deu por que a estrutura dos dados poderia promover vícios, em função de haver poucas fêmeas com número suficiente de crias com informações, ou seja, a grande maioria apresentava apenas uma cria.

Inicialmente, foram realizadas análises unicaracterística paraPN,PD,GNDePA.Posteriormente, foi realizada uma análise multicaracterísticas contendo PN, PD e GND.

\section{RESULTADOS E DISCUSSÃO}

A raça Somalis Brasileira apresenta-se como uma raça de tamanho pequeno, com um bom ganho de peso na fase pré-desmama, considerando-se as condições de manejo em pastagem nativa do semiárido Nordestino, conforme pode ser observado pelas médias apresentadas na tabela 1 . 
Tabela 1 - Número de observações $(\mathrm{N})$, médias observadas, desvio-padrão (DP) e coeficiente de variação $(\mathrm{CV})$ para as características de crescimento na raça Somalis Brasileira.

\begin{tabular}{lccc}
\hline Característica & $\mathbf{n}$ & Média \pm DP & CV\% \\
\hline Peso ao nascer $(\mathrm{kg})$ & 644 & $2,54 \pm 0,43$ & 17,04 \\
Peso ao desmame $(\mathrm{kg})$ & 419 & $14,15 \pm 3,76$ & 26,55 \\
$\begin{array}{l}\text { Ganho de peso do } \\
\begin{array}{l}\text { nascimento ao desmame } \\
\text { (kg/dia) }\end{array}\end{array}$ & 419 & $0,132 \pm 0,038$ & 29,02 \\
& 658 & $31,66 \pm 4,32$ & 13,64 \\
\hline
\end{tabular}

As herdabilidades estimadas nas análises unicaracterística foram de magnitude baixa a moderada (Tabela 2). As herdabilidades diretas de GND e PA foram as mais baixas (zero para PA), enquanto a herdabilidade materna de PD foi a maior. É importante ressaltar que o rebanho Somalis Brasileira da Embrapa Caprinos e Ovinos encontra-se fechado por muitos anos, sob seleção, sem a introdução de animais externos, pelas dificuldades para adquirir animais de criadores privados. Apesar do controle de endogamia realizado, é possível que este fato seja o responsável pela reduzida variabilidade genética observada. KARIUKI et al. (2010), trabalhando com análises unicaracterística em ovinos Dorper no Quênia, também encontraram herdabilidades diretas de baixa a moderada, variando de 0,18 a 0,36 , para características pré-desmame.

As magnitudes das herdabilidades maternas podem não ter sido elevadas, mas foram todas superiores às herdabilidades diretas, demonstrando a importância da inclusão desse efeito no modelo de análise, bem como a influência da genética da matriz nesse rebanho. Estimativas de herdabilidade materna maiores que as diretas também foram relatadas por BEHZADI et al. (2007), em ovinos Kermani no Iran.

Entretanto, alguma cautela deve ser tomada com essas estimativas, em função dos seus elevados erros-padrão, em alguns casos superiores à própria estimativa. Ressalta-se o reduzido número de observações disponíveis para as análises, o que justifica esses erros-padrão elevados. Entretanto, apesar disso, devido à inexistência de estimativas para esta raça no Brasil, estes resultados apresentam importância.

As herdabilidades encontradas foram inferiores às observadas por SILVA et al. (1994), em ovinos Somalis Brasileira, no Ceará, utilizando modelo reprodutor. É importante considerar que os modelos reprodutores tendem a superestimar os valores dos parâmetros genéticos, o que justifica os menores valores observados neste estudo. QUESADA et al. (2002) observaram valores próximos aos encontrados neste trabalho, com herdabilidade direta variando de 0,07 a 0,21 e materna variando de 0,09 a 0,22, para ovinos da raça Santa Inês, Morada Nova, e mestiços Texel x Morada Nova, no Distrito Federal.

De modo geral, a seleção massal não seria a alternativa mais indicada para promover a seleção nesse rebanho. Entretanto, a utilização de avaliações genéticas, com estimativas de diferenças estimadas na progênie (DEP), permitirá a identificação de animais superiores para promover melhoramento no rebanho. Essa prática, associada a controle de endogamia, índices de seleção e acasalamentos dirigidos, já vem sendo utilizado no rebanho. Orebanhoutiliza o Sistema de Gerenciamento de Rebanhos do GENECOC, que utiliza todos esses princípios em uma ferramenta de seleção de acasalamentos que maximizam o ganho genético e minimizam a endogamia.

Tabela 2 - Estimativas de variâncias e herdabilidade para as características de crescimento na raça Somalis Brasileira em análises unicaracterística.

\begin{tabular}{|c|c|c|c|c|c|}
\hline & $\sigma^{2}$ Genéticas & $\sigma^{2}$ Ambiente Permanente & $\sigma^{2}$ Fenotípicas & $\mathrm{h}^{2}$ & \\
\hline PN & 0,023 & $0,71 \mathrm{E}-07$ & 0,150 & PN & $0,15 \pm 0,11$ \\
\hline $\mathrm{PN}_{\mathrm{m}}$ & 0,022 & & & $\mathrm{PN}_{\mathrm{m}}$ & $0,15+0,09$ \\
\hline PD & 0,409 & 0,099 & 3,499 & PD & $0,12+0,11$ \\
\hline $\mathrm{PD}_{\mathrm{m}}$ & 0,783 & & & $\mathrm{PD}_{\mathrm{m}}$ & $0,22 \pm 0,13$ \\
\hline GND & $0,3 \mathrm{E}-04$ & $0,3 \mathrm{E}-04$ & $0,4 \mathrm{E}-03$ & GND & $0,07 \pm 0,09$ \\
\hline $\mathrm{GND}_{\mathrm{m}}$ & $0,8 \mathrm{E}-04$ & & & $\mathrm{GND}_{\mathrm{m}}$ & $0,19+0,12$ \\
\hline PA & $0,4 \mathrm{E}-04$ & 6,867 & 11,579 & PA & $0,00+0,15$ \\
\hline
\end{tabular}

$\sigma^{2}$ = variâncias; $\mathrm{h}^{2}=$ herdabilidade; $\mathrm{PN}=$ efeito direto do peso ao nascer; $\mathrm{PN}_{\mathrm{m}}=$ efeito materno do peso ao nascer; $\mathrm{PD}$ - efeito direto do peso ao desmame; $\mathrm{PD}_{\mathrm{m}}=$ efeito materno do peso ao desmame; $\mathrm{GND}=$ efeito direto do ganho de peso do nascimento ao desmame; $\mathrm{GND}_{\mathrm{m}}=$ efeito materno do ganho de peso do nascimento ao desmame; PA- efeito direto do peso adulto. 
A herdabilidade para PA foi zero (Tabela 2), demonstrando que o fenótipo não é um bom indicador do genótipo nos indivíduos estudados. Assim, esperase que a seleção individual não proporcione ganhos genéticos satisfatórios. As matrizes desse rebanho apresentam um tamanho corporal muito próximo, $\mathrm{e}$ isso pode indicar que o peso adulto da raça no rebanho está estabilizado, com reduzida possibilidade de alteração por seleção aditiva.

Pode ser observado que as variâncias genéticas das análises multicaracterísticas para PNPD-GND (Tabela 3) superaram aquelas observadas nas análises unicaracterística (Tabela 2). A maioria das herdabilidades para os efeitos genéticos direto e materno foram superiores na análise multicaracterística, com exceção para PN e GND, que obtiveram valores de herdabilidade direta de 0,04 e 0,00 (Tabela 4), enquanto na análise unicaracterística esses valores foram de 0,15 e 0,07 (Tabela 2). SOUSA et al. (1999), trabalhando com análises bicaracterísticas, relataram uma pequena redução na estimativa de herdabilidade para PN e ligeiro aumento nas estimativas para os demais pesos, quando comparadas às obtidas pelo método unicaracterística.

De um modo geral, os maiores valores de herdabilidade, na análise multicaracterística, deve-se ao melhor aproveitamento da informação disponível, permitindo o inter-relacionamento dos dados e informações, considerando a seleção praticada no período anterior, dentre outros aspectos, o que contribui para resgatar maior proporção da variância genética aditiva. SARMENTO et al. (2006) confirmaram isso em seu trabalho e sugeriram, a partir de seus resultados, que a informação de outra característica contribui na estimação das (co)variâncias.

A herdabilidade direta para GND foi igual a zero, enquanto a materna foi igual à unidade (Tabela 4), provavelmente devido ao pequeno número de dados disponíveis. As demais herdabilidades foram de moderada a alta magnitude. Chamam a atenção as altas herdabilidades, materna e direta, respectivamente, encontradas para PN e PD. SILVA \& ARAÚJO (2000), trabalhando com ovinos Santa Inês, no Ceará, também encontraram herdabilidades de moderada a alta magnitude para pesos e ganho de peso do nascimento aos 112 dias (desmame), variando de 0,36 a 0,56 .

É importante considerar que os parâmetros estimados nas análises individuais apontavam para reduzida possibilidade em explorar a seleção massal, entretanto, na análise multicaracterística, esse fato pode ser questionado. Considera-se, entretanto, que o software MTFDREML não estima errospadrão em análises multicaracterísticas, quando essas características apresentam distinto número de observações. Esse fato impede uma melhor avaliação dos parâmetros estimados. De qualquer forma, pode-se presumir que, em alguns casos, houve superestimativa do valor. Considera-se que a análise multicaracterística permitiu melhor utilização da informação e melhor avaliação da variância genética aditiva. Entretanto, o mais provável é que as estimativas sejam realmente de baixa a moderada magnitude.

Tabela 3 - Estimativas de (co) variâncias em análise multicaracterística para peso ao nascer (PN), peso ao desmame (PD) e ganho de peso do nascimento ao desmame (GND) na raça Somalis Brasileira.

\begin{tabular}{|c|c|c|c|c|c|c|}
\hline & \multicolumn{6}{|c|}{$\sigma^{2} \mathrm{e} \sigma_{\mathrm{xy}}$ Genéticas- } \\
\hline & PN & PD & GND & $\mathrm{PN}_{\mathrm{m}}$ & $\mathrm{PD}_{\mathrm{m}}$ & $\mathrm{GND}_{\mathrm{m}}$ \\
\hline PN & 0,042 & & & & & \\
\hline PD & 0,046 & 2,876 & & & & \\
\hline GND & $-0,8 \mathrm{E}-03$ & 0,031 & $0,4 \mathrm{E}-03$ & & & \\
\hline $\mathrm{PN}_{\mathrm{m}}$ & & & & 0,796 & & \\
\hline $\mathrm{PD}_{\mathrm{m}}$ & & & & $-0,057$ & 1,486 & \\
\hline \multirow[t]{3}{*}{$\mathrm{GND}_{\mathrm{m}}$} & & & & $-0,366$ & $0,1 \mathrm{E}-04$ & 0,168 \\
\hline & $\sigma_{x y}$ de $A$ & Permane & ..-..-. & $\cdots-\sigma^{2}$ e & icas----- & \\
\hline & PN & PD & GND & PN & PD & GND \\
\hline PN & 0,104 & & & 1,046 & & \\
\hline PD & & 0,290 & & $-0,011$ & 4,652 & \\
\hline GND & & & $0,1 \mathrm{E}-07$ & $-0,367$ & 0,031 & 0,169 \\
\hline
\end{tabular}

$\sigma^{2}$ = variàncias; $\sigma_{x y}=$ covariâncias; variàncias na diagonal; covariâncias fora da diagonal; $\mathrm{PN}=$ efeito direto do peso ao nascer; $P \mathrm{~N}_{\mathrm{m}}=\mathrm{efeito}$ materno do peso ao nascer; PD- efeito direto do peso ao desmame; $\mathrm{PD}_{\mathrm{m}}$ - efeito materno do peso ao desmame; GND- efeito direto do ganho de peso do nascimento ao desmame; $\mathrm{GND}_{\mathrm{m}}=$ efeito materno do ganho de peso do nascimento ao desmame.

Ciência Rural, v.43, n.5, mai, 2013. 
Tabela 4 - Estimativas, herdabilidades e correlações genéticas em análise multicaracterística para peso ao nascer (PN), peso ao desmame (PD) e ganho de peso do nascimento ao desmame (GND) na raça Somalis Brasileira

\begin{tabular}{|c|c|c|c|c|c|c|}
\hline & PN & PD & GND & $\mathrm{PN}_{\mathrm{m}}$ & $\mathrm{PD}_{\mathrm{m}}$ & $\mathrm{GND}_{\mathrm{m}}$ \\
\hline PN & 0,04 & & & & & \\
\hline PD & 0,13 & 0,62 & & & & \\
\hline GND & $-0,20$ & 0,94 & 0,00 & & & \\
\hline $\mathrm{PN}_{\mathrm{m}}$ & & & & 0,76 & & \\
\hline$P D_{\mathrm{m}}$ & & & & $-0,05$ & 0,32 & \\
\hline $\mathrm{GND}_{\mathrm{m}}$ & & & & $-1,00$ & 0,00 & 1,00 \\
\hline
\end{tabular}

Herdabilidades na diagonal; correlações genéticas fora da diagonal; $\mathrm{PN}=$ efeito direto do peso ao nascer; $\mathrm{PN}_{\mathrm{m}}=$ efeito materno do peso ao nascer; PD - efeito direto do peso ao desmame; $\mathrm{PD}_{\mathrm{m}}$ - efeito materno do peso ao desmame; GND- efeito direto do ganho de peso do nascimento ao desmame; $\mathrm{GND}_{\mathrm{m}}$ - efeito materno do ganho de peso do nascimento ao desmame.

As correlações genéticas apresentaram grande variação quanto aos seus valores (Tabela 4), desde correlações negativas, nula e positivas. As correlações entre PNxGND $(-0,20), \mathrm{PN}_{\mathrm{m}} \times \mathrm{PD}_{\mathrm{m}}(-0,05)$ e $\mathrm{PN}_{\mathrm{m}} \mathrm{xGND} \mathrm{F}_{\mathrm{m}}(-1,00)$ foram negativas, mostrando que a associação entre essas características tem sentidos opostos. Esses valores foram diferentes das demais correlações genéticas encontradas na literatura (SARMENTO et al., 2006; LOBO et al., 2009). REGO NETO et al. (2010) também encontraram correlação negativa entre o PN e o peso aos 196 dias de idade na raça Santa Inês. Correlação negativa é um indicativo de como é difícil melhorar, simultaneamente, ambas as características em um programa de seleção (NESER et al., 2001). Esses diferentes resultados encontrados podem ser explicados, parcialmente, devido aos parâmetros genéticos dependerem da formação histórica, forças seletivas e aspectos ambientais da população (LOBO et al., 2009), além das diferenças no genótipo, raça, localização e rebanho (HASSEN et al., 2003).

A correlação entre PDxGND $(0,94)$ foi alta e positiva, seguindo o que normalmente é encontrado na literatura, pois grande parte dos genes responsáveis por maiores pesos à desmama também está atuando para maiores ganhos de peso. SARMENTO et al. (2006) também encontraram correlações altas e positivas para características de crescimento em ovinos Santa Inês.

\section{CONCLUSÃO}

A análise multicaracterística apresentou superioridade em relação à unicaracterística, com melhor robustez para resgatar variância genética, por considerar as relações entre as características e por permitir maior eficiência no uso da informação disponível para o rebanho estudado. As correlações genéticas apresentaram grande variação nos seus valores.

\section{REFERÊNCIAS}

BEHZADI, M.R.B. et al. Estimates of genetic parameters for growth traits in Kermani sheep. Journal of Animal Breeding and Genetics, v.124, n.5, p.296-301, 2007. Disponível em: <http://onlinelibrary. wiley.com/doi/10.1111/j.1439-0388.2007.00672.x/pdf>. Acesso em: 20 jun. 2011. doi 10.1111/j.1439-0388.2007.00672.x.

BOLDMAN, K.G. et al. A manual for use of MTDFREML. A set of programs to obtain estimates of variances and covariances [DRAFT]. Lincoln: USDA/Agricultural Research Service, 1995. 120p.

HASSEN, Y. et al. Genetic parameter estimates for birth weight, weaning weight and average daily gain in pure and crossbred sheep in Ethiopia. Journal Animal Breeding and Genetics. v.120, p.29-38, 2003. Disponível em: <http://onlinelibrary.wiley.com/ doi/10.1046/j.1439-0388.2003.00361.x/pdf>. Acesso em: 20 jun. 2011. doi: 10.1046/j.1439-0388.2003.00361.x.

KARIUKI, C.M. et al. Direct and maternal (co)variance components, genetic parameters and annual trends for growth traits of Dorper sheep in semi-arid Kenya. Tropical Animal Health and Production, v.42, p.473-481, 2010. Disponível em: <http:// www.springerlink.com/content/qg036516730153k3/fulltext.pdf>. Acesso em: 22 jun. 2011. doi: 10.1007/s11250-009-9446-0.

LÔBO, A.M.B.O. et al. Genetic parameters for growth, reproductive and maternal traits in a multibreed meat sheep population. Genetics and Molecular Biology, v.32, n.4, p.761-770, 2009. Disponível em: <http://www.scielo.br/scielo. php?script=sci_arttext\&pid=S1415-47572009000400016\&lng=e n\&nrm=iso>. Acesso em: 17 maio, 2011. doi: 10.1590/S141547572009005000080 .

NESER, F.W.C. et al. Genetic parameter estimates for pré-weaning weight traits in Dorper Sheep. Small Ruminant, v.40, p.197-202, 2001. Disponível em: <http://www.sciencedirect.com/science/ article/pii/S0921448801001729>. Acesso em: 13 jun. 2011. doi: 10.1016/S0921-4488(01)00172-9. 
QUESADA, M. et al. Efeitos genéticos e fenotípicos sobre características de produção e reprodução de ovinos deslanados no Distrito Federal. Revista Brasileira de Zootecnia, v.31, n.1, p.342-349, 2002 (suplemeto). Disponível em: <http://www. revistasbz.org.br/scripts/revista/SBZ1/Artigos/2978.PDF>. Acesso em: 2 dez. 2010.

REGO NETO, A.A. et al. Estimação de componentes de variância e parâmetros genéticos para pesos do nascimento aos 196 dias de idade em ovinos Santa Inês por meio de modelo multicaracterística. In: REUNIÃO ANUAL DA SOCIEDADE BRASILEIRA DE ZOOTECNIA, 47., 2010, Salvador, BA. Anais... Salvador: Sociedade Brasileira de Zootecnia, 2010. 1 CD.

SARMENTO, J.L.R. et al. Estimação de parâmetros genéticos para características de crescimento de ovinos Santa Inês utilizando modelos uni e multicaracterísticas. Arquivo Brasileiro de Medicina Veterinária e Zootecnia, v.58, n.4, p.581-589, 2006. Disponível em: <http://www.scielo.br/scielo.php?script=sci_artte xt\&pid=S0102-09352006000400021>. Acesso em: 02 dez. 2010. doi: 10.1590/S0102-09352006000400020.

SAS INSTITUTE INC. SAS/STAT. User's guide, version 6.11. 4.ed. Cary, 1996. V.842p.

SCHAEFFER, L.R. Animal models: why, how and to use them. In: SIMPÓSIO NACIONAL DE MELHORAMENTO ANIMAL,
1996, Ribeirão Preto, SP. Anais... Ribeirão Preto: SBMA, 1996. p.21-40.

SILVA, F.L.R.; ARAÚJO, A.M. Características de reprodução e de crescimento de ovinos mestiços Santa Inês, no Ceará. Revista Brasileira de Zootecnia, v.29, n.6, p.1712-1720, 2000. Disponível em: <http://www.revistasbz.org.br/scripts/revista/ SBZ1/Artigos/2501.PDF>. Acesso em: 02 dez. 2010.

SILVA, F.L.R. da. et al. Parâmetros genéticos e fenotípicos dos pesos pré-desmama em ovinos da raça Somalis Brasileira, no Ceará. Revista Brasileira de Zootecnia, v.23, n.3, p.377-382, 1994. Disponível em: <http://www.revistasbz.org.br/scripts/revista/SBZ1/ Resumo.asp?NumArtigo=460>. Acesso em: 02 dez. 2010.

SILVA, F.L.R. da. et al. Desempenho produtivo e reprodutivo da raça Somalis. In: REUNIÃO TÉCNICO-CIENTÍFICA DO PROGRAMA DE APOIO A PESQUISA COLABORATIVA DE PEQUENOS RUMINANTES, 1986, Sobral, CE. Anais... Sobral: Reunião Técnico-Científica do programa de apoio a pesquisa colaborativa de pequenos ruminantes, 1986. p.347-353.

SOUSA, W.H. de. et al. Estimativas de componentes de (co) variância e herdabilidade direta e materna de pesos corporais em ovinos da raça Santa Inês. Revista Brasileira de Zootecnia, v.28, n.6, p.1252-1262, 1999. Disponível em: <http://www.revistasbz. org.br/scripts/revista/SBZ1/Artigos/2478.PDF>. Acesso em: 02 dez. 2010. 